mit den Standardmaßnahmen allein. Auch bei Semaglutid, einem noch potenteren GLP-1-Rezeptor-Agonisten, sehen wir diesen Effekt.

Das ist das das einmal wöchentlich anwendbare Inkretinmimetikum? Wie weit ist es in der Entwicklung?

Krogsgaard: Ja. Die injizierbare Form ist in der fortgeschrittenen Phase 3, für die orale ist die klinische Phase 2 gerade abgeschlossen. Mit einer Injektion von $1 \mathrm{mg}$ pro Woche in Monotherapie konnte das $\mathrm{HbA}_{1 \mathrm{c}}$ von $7,9 \%$ auf $6,0 \%$ gesenkt werden bei $6,5 \mathrm{~kg}$ Gewichtsverlust nach 26 Wochen. Das sind noch einmal 0,6\%-Punkte und 3,5 kg Gewicht weniger als mit Liraglutid. Die stärkste $\mathrm{HbA}_{1 \mathrm{c}}$-Absenkung die ich je mit einem Basalinsulin gesehen habe, war auf 6,6\%, und dann haben die Patienten häufig Hypoglykämien.

Glauben Sie es wird einmal eine etablierte medikamentöse Diabetesprophylaxe geben, vergleichbar mit der Lipidsenkung in Bezug auf Herzinfarkte?

Krogsgaard: Ich würde es gern glauben. Vielleicht dann, wenn es uns gelingt, eine Hochrisikopopulation mit bestimmten Komorbiditäten und erhöhter Sterblichkeit auszumachen wie bei den PCSK-
9-Hemmern. Bisher wissen wir auch noch nicht, ob wir den Ansatz mit Liraglutid oder Semaglutid weiter verfolgen werden.

Last but not least findet sich auf diesem Kongress eine Reihe von Postern zum faster-acting Insulin Aspart. Wann ist damit auf dem Markt zu rechnen?

Krogsgaard: Wir sind sehr zufrieden mit den Ergebnissen, weil der postprandiale Blutzucker damit um mehr als $1 \mathrm{mmol} / \mathrm{l} \mathrm{mehr}$ gesenkt wurde als mit NovoRapid ${ }^{\circledR}$. Das war genug, um den $\mathrm{HbA}_{1 \mathrm{c}}$-Wert bei Typ-1-Diabetikern signifikant zu reduzieren. Besonders in der Studie zur Insulinpumpentherapie sind die Daten sehr schön [5]. Wenn alles wie geplant vorangeht, werden wir es hoffentlich um den Jahreswechsel 2016/2017 in den ersten Märkten einführen können.

Interview: Sarah L. Pampel

\section{Quellen:}

1. Buse et al. Diabetes 2015;Vol. 64, Suppl 1, A43. Oral 166

2. Warren et al. Diabetes 2015;Vol. 64, Suppl 1, A266. Poster 1040

3. Dejgaard et al. Diabetes 2015;Vol. 64, Suppl 1, A73. Oral 277

4. Frandsen et al. Diabetes 2015;Vol. 64, Suppl 1, A74. Oral 279

5. Bode et al. Diabetes 2015;Vol. 64, Suppl 1, A253, Poster 994

\title{
Doch keine kardiovaskuläre Risikoerhöhung durch Sulfonylharnstoffe?
}

Die Ergebnisse einer neuen Metaanalyse, die 47 randomisierte kontrollierte Studien mit 37650 Patienten berücksichtigte, spricht gegen eine kardiovaskuläre Risikoerhöhung durch Sulfonylharnstoffe.

Sulfonylharnstoffe sind eine sehr preisgünstige Alternative zu moderneren oralen Antidiabetika, wenn der Typ-2-Diabetiker neben Metformin ein weiteres Medikament benötigt. Bekannte Nachteile, etwa gegenüber DPP-4-Hemmer oder SGLT-2-Inhibitoren, sind das Hypoglykämie-Risiko sowie die Gewichtszunahme. Seit einiger Zeit wird darüber hinaus debattiert, ob die antidiabetischen Evergreens das kardiovaskuläre Risiko erhöhen. Eine Reihe von Metaanalysen haben widersprüchliche Ergebnisse geliefert.

\section{Risikoerhöhung in Kohortenstudien}

2013 hatte eine deutsche Autorengruppe um T. Forst und M. Hanefeld in der Zeitschrift Diabetes and Vascular Disease Research (Diab Vasc Dis Res. 2013 Jul;10(4):302-14) eine Meta-Analyse von 20 Kohorten- und Beobachtungsstudien mit mehreren $100000 \mathrm{~Pa}$ tienten publiziert. Die Autoren fanden - allerdings bei sehr heterogenem Patientenkollektiv - nahezu eine Verdopplung der Gesamtmortalität von Patienten, die Sulfonylharnstoffe einnahmen.

\section{Keine Risikoerhöhung in randomisierten Studien}

Brasilianische Forscher um D. V. Rados vom Hospital de Clinic in Porto Alegre stellten beim Jahreskongress der American Diabetes Association 2015 in Boston nun eine neue Metaanalyse vor, die mit 47 Studien und zusammen 37650 Patienten zwar eine kleinere Datenbasis aufweist als die deutsche Metaanalyse. Dafür hatten die Brasilianer ausschließlich randomisierte und kontrollierte Studien analysiert und damit nicht nur einen komplett anderen Datensatz, sondern auch höherwertige Studien.

Sie fanden keine signifikante Risikoerhöhung für die Gesamtmortalität (OR: 1,12), für die kardiovaskuläre Mortalität (OR: 1,12), für den Herzinfarkt oder für den Schlaganfall. Nach ihrer Einschätzung ist die Evidenz bezüglich der Mortalität sehr gut und bezüglich Herzinfarkt und Schlaganfall moderat. Bei Analyse einzelner Sulfonylharnstoffe fanden sie, dass nur das in Deutschland nicht verbreitete Glipizid mit einem erhöhten Sterberisiko verbunden war. Solche Subgruppen-Analysen seien jedoch mit Vorsicht zu betrachten.

\section{Post-hoc-Analyse der SAVOR-Studie findet kein Risikosignal für Sulfonylharnstoffe}

Ähnliche Befunde berichten Autoren einer Post-hoc-Analyse der SAVOR-TIMI-53-Studie, in der die Sicherheit des DPP4-Hemmers Saxagliptin im Vergleich zu anderen Antidiabetika untersucht und bestätigt worden war. Sie hatten überprüft, ob die Studienpatienten, die mit Sulfonylharnstoffen (SU) behandelt wurden, mehr kardiovaskuläre Ereignisse erlitten als Patienten ohne SU. Dies war nicht der Fall. Unter insulinpflichtigen Diabetikern hatte eine Therapie mit SU sogar einen deutlichen Schutzeffekt vor Krankenhauseinweisungen wegen instabiler Angina pectoris ( $0,3 \%$ vs. $1,6 \%)$.

Dr. med. Dirk Einecke

Quelle: American Diabetes Association, 75. Scientific Sessions, Boston, 5.-9. Juni 2015 